\title{
Принцип «Damage control» в хирургии острой спинальной травмы
}

\author{
Ю. Е. Педаченко, А. С. Нехлопочин \\ Институт нейрохирургии имени академика А. П. Ромоданова НАМН Украины, г. Киев

\section{The principle of «Damage control» in surgery of an acute spinal trauma}

\author{
Yu. E. Pedachenko, A. S. Nekhlopochin
}

Romodanov Institute of Neurosurgery, Kiev

\begin{abstract}
Реферат
Цель исследования. Анализ эффективности применения подхода двухэтапного Damage Control в терапии позвоночно-спинномозговой травмы у пациентов на фоне тяжелой политравмы (ПТ).

Материалы и методы. Проведен ретроспективный анализ результатов терапии у 53 пациентов. В зависимости от сроков и объема проведенного хирургического лечения пациенты разделены на три группы. Анализировались показатели регресса неврологических расстройств, летальности и длительности интенсивной терапии.

Результаты. Пациенты, которым выполнялось отсроченное хирургическое вмешательство, имели наихудшие показатели восстановления неврологического дефицита. Раннее одномоментное декомпрессивно-стабилизирующее вмешательство обеспечивало лучший регресс неврологических расстройств, однако характеризовалось достаточно высокой летальностью и длительностью интенсивной терапии. В группе пациентов, у которых проводилось этапное хирургическое лечение, отмечены наилучшие значения анализируемых показателей.

Выводы. Тактика Damage Control обеспечивает наилучшие результаты терапии позвоночно-спинномозговых повреждений у пациентов на фоне политравмы.

Ключевые слова: спинальная травма; хирургия позвоночника; стабилизация; хирургическое лечение; декомпрессия.

Abstract

Objective. Analysis of efficacy of the two-staged Damage Control approach application in treatment of vertebro-spinal trau$\mathrm{ma}$ in patients on background of severe polytrauma.

Materials and methods. Retrospective analysis of the treatment results in 53 patients was conducted. Depending on terms and volume of the surgical treatment conducted the patients were divided on three groups. The indices of the neurological disorders regress, lethality and the intensive therapy duration were analyzed.

Results. The patients, to whom delayed surgical intervention was conducted, have had the worst indices of the neurological deficiency restoration. The early one-stage decompressive-stabilizing intervention have guaranteed better regress of neurological disorders, but was characterized by certainly high lethality and enhanced intensive therapy duration. In this group of patients, in whom the staged surgical treatment was conducted, best values of the analyzed indices were noted.

Conclusion. The Damage Control tactics provides best results of treatment of vertebro-spinal injuries in patients on background of polytrauma.

Keywords: spinal trauma; vertebral surgery; stabilization; surgical treatment; decompression.
\end{abstract}

Исторически применительно к травме опорно-двигательного аппарата в объеме ПТ изначально использовали принцип «слишком больной, чтобы быть прооперированным» (англ. - «too sick to operate on»), регламентирующий возможность проведения отсроченного до 14 дней оперативного лечения. В дальнейшем, учитывая качество жизни пациентов, перенесших ПТ, социальные и экономические составляющие, стали применять концепцию «слишком больной, чтобы не быть прооперированным» (англ. - «too sick not to operate on»), определяющую восстановление функции как ведущий критерий эффективной терапии, несмотря на более высокий риск для пациента [1]. Этот принцип, трансформировавшийся в достаточно известный «Early total care» (ETC), фактически использовали в большинстве протоколов до 2000 Г.

В настоящее время в оказании помощи пациентам с тяжелой ПТ, как в полостной хирургии, так и в ортопедии, применяют принцип «Damage control surgery» (DC-S), регламентирующий выполнение оперативных вмешательств в острейшем периоде травмы в объеме, достаточном для предотвращения осложнений, угрожающих жизни пациента, и исключающем развитие стойких значительных функциональных нарушений, но не реализующем выполнение хирургической задачи в полном объеме. Однако ряд тактических вопросов хирургии спинальной травмы у пациентов с тяжелой ПТ остаются дискутабельными и требуют дальнейшего изучения.

Цель исследования: анализ эффективности двухэтапного DC-S в терапии позвоночно-спинномозговой травмы у пациентов с тяжелой ПТ.

\section{Материалы и методы исследования}

Проведен ретроспективный анализ результатов лечения пациентов с ПТ в период с января 2015 по декабрь 2016 года.

Критерии формирования выборки:

- степень тяжести ПТ по шкале Injury Severity Score (ISS) соответствовала диапазону 17 < ISS; 
- нестабильное повреждение позвоночника, верифицированное с помощью спиральной компьютерной томографии, с явлениями компрессии позвоночного канала и требующее оперативного вмешательства;

- неврологический дефицит, соОтветствующий группам A-C по ASIA scale;

- длительность наблюдения 3 - 45 сут.

В зависимости от сроков и объема оказания специализированной нейрохирургической помощи пациенты были разделены на группы:

- пациенты, первично госпитализированные в районные ЛПУ, хирургическое лечение которых было отложено до относительной стабилизации соматического статуса, т.е. фактически проводилась терапия по принципу «too sick to operate on»;

- пациенты, первично госпитализированные в специализированное ЛПУ, помощь которым была оказана своевременно и в полном объеме в соответствии с действующими протоколами (принцип «Early total care»);

- пациенты, первично госпитализированные в специализированное ЛПУ, оказание нейрохирургической помощи которым проводилось поэтапно в соответствии с принципами DC-S.

Оценка однородности выборки. Принимая во внимание, что шкала ISS имеет преимущественно анатомическую направленность и скорее скрининговый характер, с целью более эффективной оценки тяжести травмы всех анализируемых пациентов, мы использовали комбинированную анатомо-физиологическую шкалу Trauma Injury Severity Score (TRISS) [2], которая представлена тремя субшкалами: Revised Trauma Score (RTS), ISS и шкалой оценки возраста пациента, а также коэффициентами для тупой и проникающей травмы.

Расчет RTS: RTS $=(0.9368 \times$ GCS Value $)+(0.7326 \times$ SBP Value $)+(0.2908 \times$ RR Value $)$, где GCS Value, SBP Value и RR Value - баллы соответствия параметрам шкалы комы Глазго, систолическому АД и частоте дыхания.

Расчет значения TRISS:

$$
\text { TRISS }=\frac{1}{\left(1+\mathrm{e}^{-(-0,76+1,03 \times \text { RTS }-0,07 \times \text { ISS }-0,04 \times \text { AgeIndex })}\right)},
$$

где е $=2,71828$.
Коэффициенты для тупой травмы, использованные в нашем исследовании, соответствуют приведенным в авторской публикации [3] и достоверно отражают уровень летальности. Пациенты с проникающей травмой в наше исследование не включались.

Оценка эффективности терапии. С целью определения эффективности и целесообразности анализируемых тактических подходов в терапии осложненной позвоночно-спинномозговой травмы в комплексе лечения пациентов с ПТ были использованы следующие критерии.

1. Динамику восстановления неврологических расстройств оценивали с использованием схемы International Standards For Neurological Classification of Spinal Cord Injury (ISNCSCI) [3]. Исходным уровнем неврологического статуса служили данные, описанные в момент поступления. В качестве конечных значений использовали данные неврологического статуса при выписке пациента либо, в случае летального исхода, зарегистрированные за сутки до констатации биологической смерти.

Так как в исследовании рассматривали пациентов с различным уровнем травмы, с целью унификации полученных результатов рассчитывали среднее значение двигательной активности и состояние чувствительной сферы в сегментах ниже уровня травмы. По разнице финальных и исходных показателей судили о динамике восстановления.

2. Общий уровень летальности, который рассчитывался как доля от всех анализируемых пациентов каждой группы на протяжении всего периода пребывания в стационаре вне зависимости от причины, непосредственно приведшей к летальному исходу.

3. Длительность проведения интенсивной терапии. В случае госпитализации пациента в районную больницу длительность пребывания в отделении интенсивной терапии также учитывали при расчете показателя. В случае летального исхода данные длительности пребывания пациента в ОРИТ не учитывали при расчете среднего показателя для группы.

Статистическая обработка данных. Анализ однородности анализируемых групп пациентов определяли с применением критерия Краскела-Уоллиса.

\begin{tabular}{|c|c|c|c|c|}
\hline \multicolumn{5}{|c|}{ Основная характеристика групп пациентов } \\
\hline Параметры & $\begin{array}{c}\text { LTC } \\
(n=18)\end{array}$ & $\begin{array}{c}\text { ETC } \\
(n=20)\end{array}$ & $\begin{array}{c}D C \\
(n=15)\end{array}$ & $\mathrm{p}$ \\
\hline Возраст, лет ( $\bar{x} \pm m)$ & $37,28 \pm 3,36$ & $37,6 \pm 2,94$ & $38,73 \pm 3,99$ & 0,9953 \\
\hline Пол, м/ж & $15 / 3$ & $16 / 4$ & $12 / 3$ & 0,9578 \\
\hline Время до операции, ч ( $\bar{x} \pm m)$ & $134,0 \pm 8,99$ & $4,83 \pm 0,37$ & $4,77 \pm 0,36$ & $0,9125 *$ \\
\hline \multicolumn{4}{|l|}{ Обстоятельства травмы: } & \multirow{5}{*}{0,7963} \\
\hline дтп & 4 & 5 & 4 & \\
\hline падение с высоты & 7 & 6 & 2 & \\
\hline травма на производстве & 5 & 7 & 6 & \\
\hline другое & 2 & 2 & 3 & \\
\hline ISS $(\bar{x} \pm m)$ & $32,98 \pm 1,69$ & $32,55 \pm 1,65$ & $32,27 \pm 1,26$ & 0,931 \\
\hline Группа ASIA, A/B/C & $6 / 8 / 4$ & $7 / 8 / 5$ & $5 / 6 / 4$ & 0,9987 \\
\hline
\end{tabular}




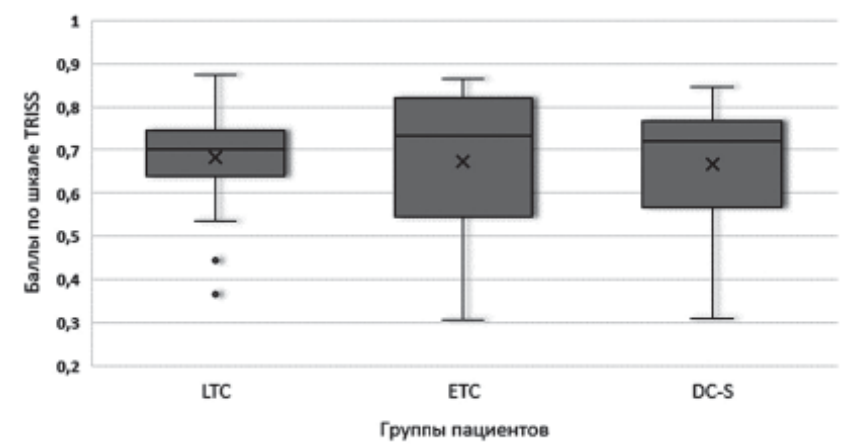

Степень тяжести ПТ у пащиентов анализируемъх групп.

Принадлежность полученных показателей нормальному закону распределения проверяли с использованием критерия Колмогорова-Смирнова. При нормальном распределении величин использовали критерий Стьюдента для независимых групп, в остальных случаях - непараметрический U-критерий Манна - Уитни. Качественные параметры анализировали с применением критерия согласования Пирсона.

\section{Результаты}

На основании оценки данных, полученных из историй болезней за анализируемый период, отобраны 53 пациента, которые удовлетворяли критериям выборки.

Характеристика групп пациентов

В зависимости от тактики хирургического вмешательства выделены три группы: позднее хирургическое лечение (Late Total Care, LTC) - 18 пострадавших; раннее хирургическое лечение (Early Total Care, ETC) - 20 пациентов; хирургическое лечение по принципам DC-S - 15 пострадавших (см. таблииу).

Проанализированы характер и степень тяжести повреждений у пациентов в зависимости от анатомической области.

Основным критерием, по которому определяли степень тяжести ПТ, послужило значение TRISS (cм. рисуHOK)).

Как следует из приведенных данных, группы пациентов были практически идентичны по степени тяжести ( $\mathrm{p}=0,9523)$. Так, значение TRISS для группы LTC coставило 0,6818 $\pm 0,0321(0,3656-0,8747)$, ETC - 0,6727 \pm $0,0398(0,3062-0,8660)$ и DC-S - 0,6664 $\pm 0,0397(0,3097$ $-0,8466)$.

Оченка результатов лечения

Анализ регресса неврологической симптоматики у пациентов при различных тактических подходах к терапии спинальной травмы выявил значительные отличия.

Результаты проведенных исследований показали, что наименьшая динамика в результате лечения достигнута у пациентов, перенесших позднее хирургическое вмешательство: нарастание двигательной активности составило $(0,1752 \pm 0,0459)$ балла, что соответствовало 3,5\%. Различия с предоперационным периодом достоверны $(\mathrm{p}=0,001)$.

Исходом лечения в пределах анализируемого срока наблюдения явилась моторная активность в объеме $(0,4109 \pm 0,1022)$ балла (8,22\% от нормы).
При раннем хирургическом лечении по принципу «Early Total Care» наблюдали значительно более выраженный регресс двигательных расстройств ( $=0,0002)$. Динамика в сравнении с предоперационным периодом составила $(0,8133 \pm 0,1368)$ балла $(16,27 \%)$, что позволило достигнуть среднего значения моторной активности в сегментах ниже уровня травмы в объеме $25,28 \%$ от нормы - $(1,2641 \pm 0,1722)$ балла. Отличия анализируемого показателя с предоперационным периодом достоверны $(\mathrm{p}=0,00001)$.

Наибольший регресс нарушений моторики отмечен у пациентов, которых лечили по принципам DC-S: объем моторных функций в сегментах ниже уровня поражения спинного мозга составил $(1,7529 \pm 0,2452)$ балла, или 35,06\% от нормального показателя. Средний прирост анализируемого значения - $(1,3104 \pm 0,1813)$ балла (26,21\%). Отличия достоверны как в сравнении с предоперационными показателями ( $<<0,00001)$, так и с группой $\operatorname{ETC~}(p=0,032)$.

Принимая во внимание, что спинной мозг является анатомически единой структурой, вполне закономерно, что динамика регресса расстройств различных сфер функциональной активности имела некоторое сходство.

Статистически наблюдали умеренную корреляцию показателей восстановления двигательных и чувствительных нарушений ( $\mathrm{r}=0,632$ для доверительного интервала 95\%).

Отмечена разница до- и послеоперационных средних значений чувствительной сферы в сегментах ниже уровня травмы.

Аналогично чувствительной сфере, наименее значимую динамику восстановления наблюдали у пациентов группы LTC. Регресс сенсорных расстройств в сегментах ниже уровня травмы составил $(0,0733 \pm 0,0207)$ балла, что соответствовало 3,67\%. Общее состояние чувствительной сферы к концу наблюдения у пациентов группы LTC было равно 16,23\% от нормы - (0,3245 \pm 0,0957) балла. Отличия с предоперационным периодом достоверны $(\mathrm{p}<0,0001)$.

При анализе динамики регресса сенсорных расстройств в группах ETC и DC-S статистически достоверной разницы не зарегистрировано ( $\mathrm{p}=0,2357)$. В группе ЕТС улучшение чувствительности составило $(0,2034 \pm$ 0,0329) балла, что соответствует $10,17 \%$, в группе DC-S $(0,2629 \pm 0,0363)$ балла $(13,15 \%)$. Итоги терапии по анализируемому показателю для групп ЕTC и DC-S эквивалентны: соответственно $(0,3685 \pm 0,0404)$ балла $(18,43 \%$ от нормы) и $(0,4594 \pm 0,0565)$ балла $(22,97 \%$ от нормы). Полученные результаты в обеих группах статистически достоверно отличаются от предоперационных показателей $(\mathrm{p}<0,0001)$.

Анализ летальности в группах пациентов свидетельствует о достаточно убедительном преимуществе DC хирургического подхода в тактике лечения ПТ. Летальность в группе DC-S составила 13,3\% от общего количества пациентов. Наибольший показатель летальности зарегистрирован в группе ЕТС - 30\%. У пациентов группы LTC этот показатель соответствовал 22,2\%. 
На основании оценки средней продолжительности пребывания пациентов в отделении интенсивной терапии получена следующая картина. Наибольшие сроки, зарегистрированные в группе LTC, составили $(262,71 \pm$ $15,26)$ ч. Следует отметить, что в указанной группе показатель рассчитан как сумма длительности пребывания в ОРИТ районных ЛПУ при первичной госпитализации и ОРИТ Луганской областной клинической больницы.

В группе ЕСТ анализируемый показатель составил $(171,29 \pm 14,71)$ ч, что достоверно меньше, чем в предыдущей группе ( $\mathrm{p}=0,0147)$. Наименьший срок пребывания в отделении интенсивной терапии отмечен в группе DC-S - $(129,15 \pm 12,77)$ ч. У 7 больных после повторного оперативного вмешательства в течение определенного промежутка времени - $(13,71 \pm 2,67)$ ч потребовалась интенсивная терапия в специализированном отделении, что также учтено при анализе. По данному показателю отличия достоверны и от группы ETC ( $\mathrm{p}=0,0416)$, и от группы LTC $(\mathrm{p}<0,0001)$.

\section{Обсуждение}

Результаты исследования в целом коррелируют с данными ряда авторов, полученными при анализе терапии тяжелой позвоночно-спинномозговой травмы. Абсолютно закономерным представляется тот факт, что ранняя декомпрессия спинного мозга является одним из наиболее важных критериев, определяющих динамику и объем восстановления неврологических функций. На основании данных мультицентрового исследования, выполненного в Канаде, J. R. Wilson и соавторы убедительно продемонстрировали лучший объем восстановления неврологических функций у пациентов, декомпрессия спинного мозга которым было проведена в течение первых 24 часов с момента травмы [5]. Аналогичные данные приводят и другие авторы [6]. В настоящее время достаточно подробно изучены и описаны цитологические механизмы развития травматической болезни спинного мозга, обратимость которых зачастую определяется длительностью механического воздействия.

В итоге проведенного исследования получены данные, достаточно убедительно иллюстрирующие преимущество принципа DC-S для восстановления неврологических функций. При анализе литературы мы не нашли публикаций, подтверждающих либо опровергающих полученные нами данные. Однако ряд авторов отмечает, что на степень восстановления неврологических функций достоверно влияют такие показатели, как уровень артериального давления, показатели гемоглобина, стабильность кислотно-основного состояния, длительность проведения искусственной вентиляции легких и использование адреномиметических препаратов [6]. В этом аспекте DC-S имеет явные преимущества за счет минимизации хирургического воздействия на пациентов с ПТ.

Уровень летальности в нашем исследовании несколько отличается от данных, опубликованных в специальной литературе. Так, D. Lubelski и соавторы сообщают о летальности у пациентов с ПТ при ранней стабилизации на уровне 6\%, при поздней - 21\% [7]. Эти данные приводятся на основании ретроспективного анализа пациентов, находившихся на лечении в MetroHealth Medical Center, Cleveland, Ohio. Как в указанном, так и в аналогичных исследованиях выполнялся анализ всех пациентов с ПТ, удовлетворяющих определенным критериям выборки. В нашем же случае позднее хирургическое вмешательство являлось результатом невозможности транспортировки пострадавших в специализированное ЛПУ, поэтому в группу LTC вошли только те пациенты, состояние которых стабилизировалось и позволило продолжить терапию с последующим хирургическим лечением. Этот факт безусловно оказывает влияние на соотношение показателей летальности в анализируемых группах пациентов.

Показатели летальности, полученные в группе DC-S, закономерно соответствуют сведениям, описанным в литературе [8]. Минимизация хирургической травмы, с одной стороны, и отсутствие риска дислокации костных фрагментов при нестабильном повреждении позвоночника, с другой, безусловно, способствуют стабилизации жизненных функций и облегчают уход за пациентами, что является основополагающим фактором профилактики осложнений у пациентов с ПТ.

Анализ причин летального исхода показал, что в группе LTC превалировали гнойно-воспалительные осложнения, в частности сепсис и пневмония, в группах ЕTC и DC-S - полиорганная недостаточность и тромбоэмболические осложнения, являющиеся последствием интенсивного травмирующего воздействия. Полученные результаты согласуются с данными других авторов [9].

Длительность пребывания пациентов в отделении интенсивной терапии убедительно свидетельствует о преимуществах этапного хирургического лечения травматических повреждений позвоночника, сочетающихся с травмой спинного мозга, у пациентов с ПТ. Закономерно высоким является показатель у пострадавших группы LTC, что обусловлено необходимостью транспортировки пациента в специализированное ЛПУ. Преимущества этапного хирургического лечения пациентов группы DC-S по сравнению с группой ETC подтверждают целесообразность подхода DC-S для минимизации вторично травмирующего хирургического воздействия и максимально ранней стабилизации витальных функций.

\section{Выводы}

1. Полученные результаты исследования достаточно убедительно и статистически достоверно демонстрируют преимущество этапного хирургического лечения неврологически осложненных травматических повреждений позвоночника у пациентов с ПТ согласно принципам DC-S.

2. Предлагаемый тактический подход позволяет достигнуть более предпочтительных показателей регресса неврологических расстройств, минимизировать уровень летальности и ускорить восстановление жизненных функций у пациентов. 


\section{References}

1. Rösch M, Klose T, Leidl R, Gebhard F, Kinzl L, Ebinger T. Cost analysis of the treatment of patients with multiple trauma. Unfallchirurg. 2000 Aug;103(8):632-9.

2. Boyd CR, Tolson MA, Copes WS. Evaluating trauma care: the TRISS method. Trauma Score and the Injury Severity Score. J Trauma. 1987 Apr;27(4):370-8.

3. Kirshblum SC, Burns SP, Biering-Sorensen F, Donovan W, Graves $\mathrm{DE}$, Jha $\mathrm{A}$, et al. International standards for neurological classification of spinal cord injury (revised 2011). J Spinal Cord Med. 2011 Nov;34(6):535-46. doi: 10.1179/204577211X13207446293695.

4. Wilson JR, Singh A, Craven C, Verrier MC, Drew B, Ahn H, et al. Early versus late surgery for traumatic spinal cord injury: the results of a prospective Canadian cohort study. Spinal Cord. 2012 Nov.8;50(11):8403.

5. Biglari B, Child C, Yildirim TM, Swing T, Reitzel T, Moghaddam A. Does surgical treatment within 4 hours after trauma have an influence on neurological remission in patients with acute spinal cord injury?
Ther Clin Risk Manag. 2016 Aug;12:1339-46. doi: 10.2147 / TCRM. S108856. eCollection 2016.

6. Ahuja CS, Schroeder GD, Vaccaro AR, Fehlings MG. Spinal Cord Injury-What Are the Controversies? J Orthop Trauma. 2017 Sep;31:S7-13. doi: 10.1097/BOT.0000000000000943.

7. Lubelski D, Tharin S, Como JJ, Steinmetz MP, Vallier H, Moore T. Surgical timing for cervical and upper thoracic injuries in patients with polytrauma. J Neurosurg Spine. 2017 Dec;27(6):633-7. doi: 10.3171/2017.4.SPINE16933. Epub 2017 Oct 6.

8. Stahel PF, VanderHeiden T, Flierl MA, Matava B, Gerhardt D, Bolles $\mathrm{G}$, et al. The impact of a standardized "spine damage-control" protocol for unstable thoracic and lumbar spine fractures in severely injured patients. J Trauma Acute Care Surg. 2013 Feb;74(2):590-6. doi: 10.1097/ TA.0b013e31827d6054

9. Konieczny MR, Strüwer J, Jettkant B, Schinkel C, Kälicke T, Muhr G, et al. Early versus late surgery of thoracic spine fractures in multiple injured patients: is early stabilization always recommendable? Spine J. 2015 Aug 1;15(8):1713-8. doi: 10.1016/j.spinee.2013.07.469. 